Trauma Berufskrankh 2005 · 7[Suppl 1]:S65-S68 DOI 10.1007/s10039-004-0956-8

Online publiziert: 5. November 2004

(c) Springer Medizin Verlag 2004
F.-O. Reiter · T. J. Hockertz · A. Gruner · H. Reilmann

Unfallchirurgische Klinik, Städtisches Klinikum, Braunschweig

\section{Lassen sich Korrekturverluste bei winkelstabilen Osteosynthesen vermeiden?}
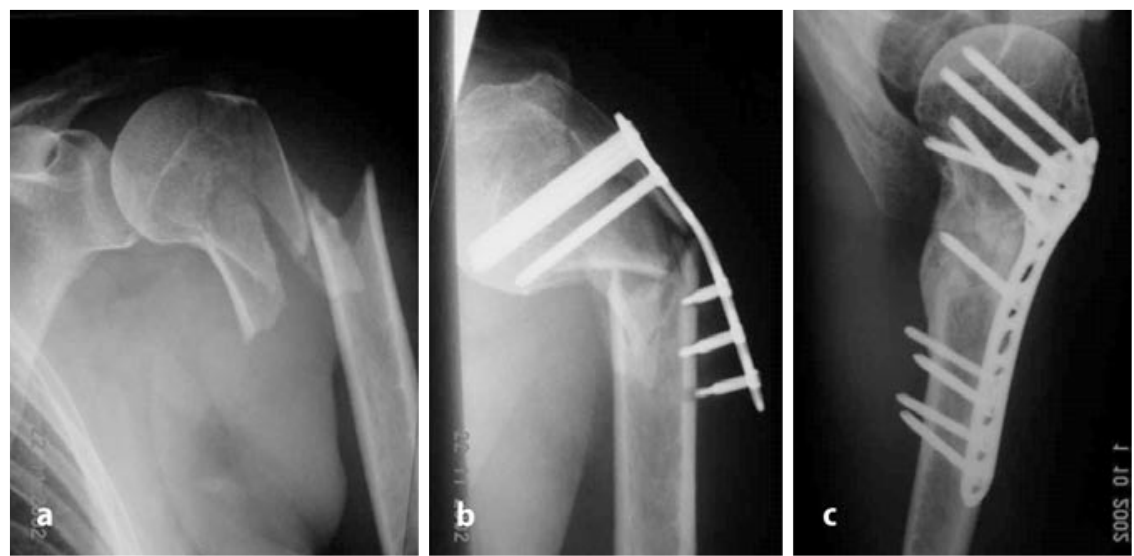

Abb. $1 \Delta$ Komplikation bei proximaler Humerusfraktur, a Initialversorgung mit 3,5-mm-Radius-TPlatte, $b$ Implantatversagen mit Ausriss der distalen Schrauben, $c$ Reosteosynthese mit Löffelplatte
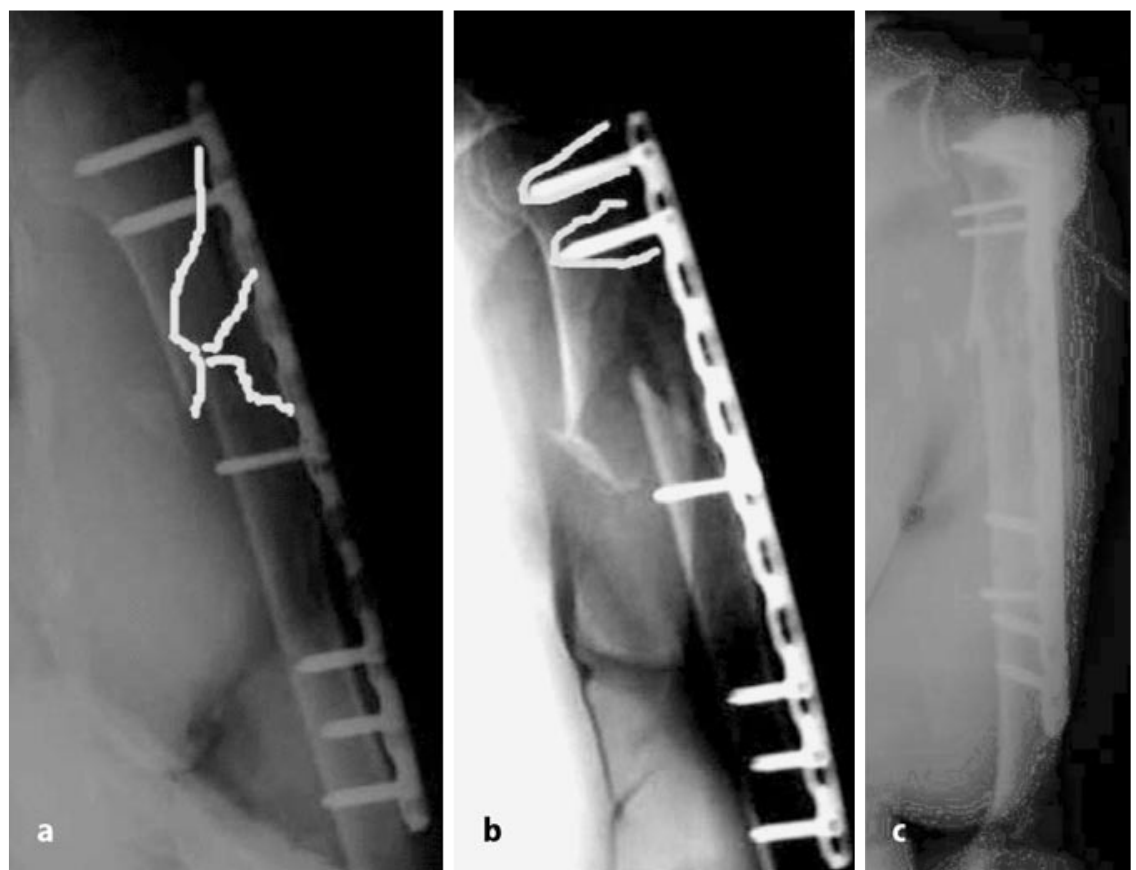

Abb. $2 \triangle$ Komplikation Humerusfraktur, a Initialversorgung mit LCP-Implantat, weiße Linien Frakturlinien, b Ausschlagen der proximalen Schrauben, bei Dislokation der Fragmente Schrauben weiterhin in der Platte verriegelt, weiße Linien zusätzlicher, durch die Instabilität der Osteosynthese erzeugter Knochendefekt, c Reosteosynthese mit winkelstabilem Implantat 

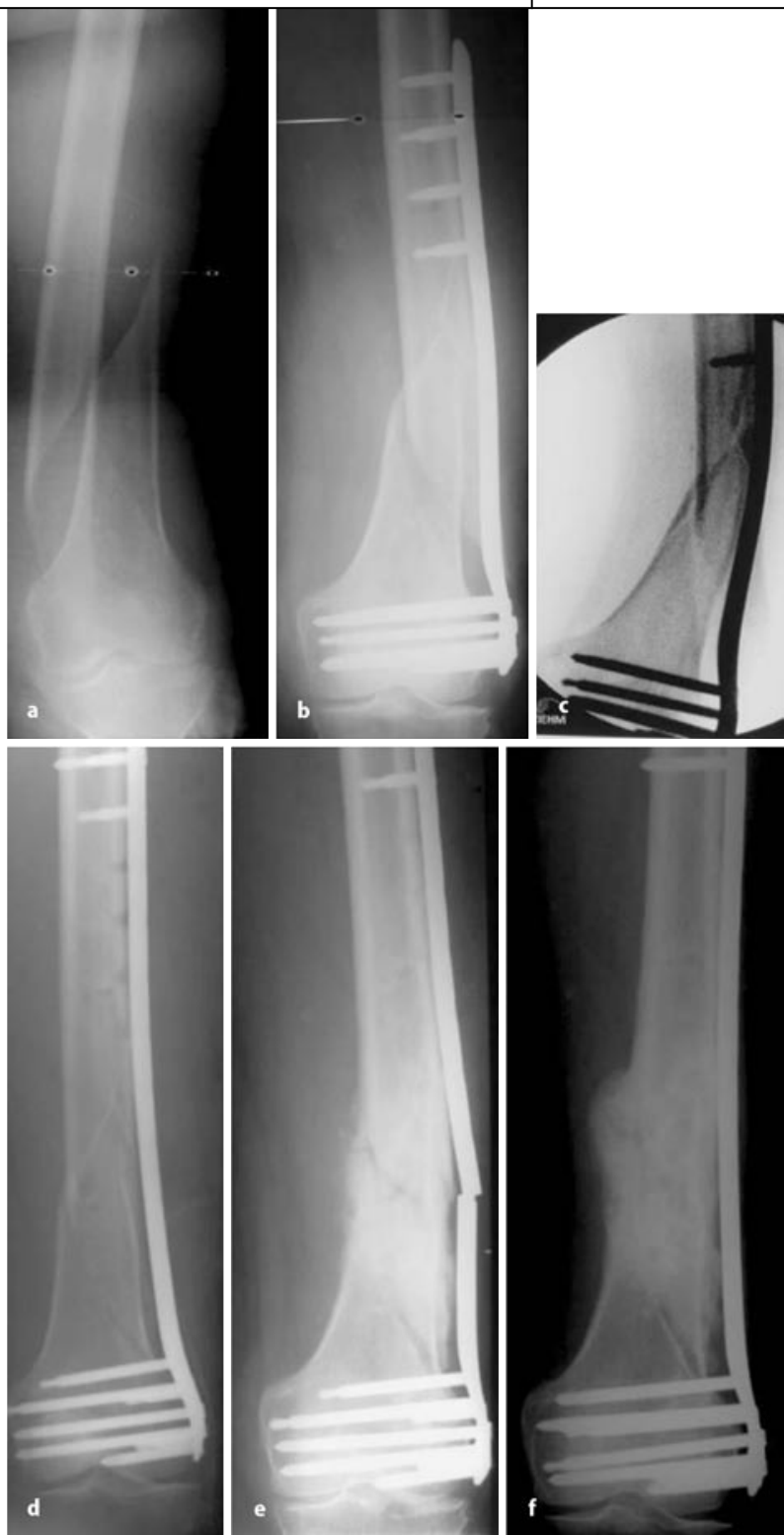

Abb. $3 \Delta$ Komplikation distale Femurfraktur, a Fraktur, b Versorgung mit LISS-Implantat, C Deformierung des Implantats unter Belastung mit Korrekturverlust, $d$ Reosteosynthese mit längerem LISS-Implantat, e erneutes Implantatversagen mit Plattenbruch. f Ausheilung nach erneuter Reosteosynthese mit LISS-Implantat

den. Er ist grundsätzlich als sekundäres Ereignis nach einer primär durchgeführten Osteosynthese zu verstehen und unabhängig vom primär erzielten Operationsergebnis in Bezug auf Gelenkrekonstruktion sowie Wiederherstellung von Achse, Länge und Rotation zu analysieren.

\section{> “...experts indicated that mecha- nical complications arose entire- ly from technical errors of appli- cations [12]".}

\section{Retrospektive Analyse}

Im eigenen Krankengut wurde eine retrospektive Analyse an 491 Osteosynthesen mit winkelstabilen Implantaten durchgeführt. Eine Übersicht über die Art der durchgeführten Osteosynthesen gibt $\bullet$ Tabelle 1. Die gesamte Komplikationsrate und die sekundären Korrekturverluste sind in $\bullet$ Tabelle 2 aufgezeigt.

Unter Betrachtung der sekundären Korrekturverluste ergibt eine Fehleranalyse anhand dieser Untersuchung folgende Ursachen:

\section{Operationstechnische Fehler - Falsche Implantatwahl \\ Unzureichende Verankerung/ Fragmentstabilisierung}

Fall 1. Eine proximale Humerusfraktur wurde initial mit 3,5-mm-Radius-T-Platte versorgt (• Abb. 1a). Im Verlauf kam es zum Implantatversagen mit Ausriss der distalen Schrauben (• Abb. 1b), da die Radius-T-Platte nur monokortikal und kurzstreckig und damit unzureichend im distalen Fragment eines strukturschwachen Knochens verankert war. Die Reosteosynthese wurde mit der Löffelplatte durchgeführt (• Abb. 1c), die für diese Art von Frakturen entwickelt wurde. Das distale Fragment wurde mit 4 bikortikalen Schrauben sicher erfasst.

Fall 2. Die Humerusfraktur der 85-jährigen Patientin wurde initial mit LCP-Implantat versorgt ( $\bullet$ Abb. 2a), wobei insgesamt nur 11/2 Schrauben sicher im proximalen Fragment verankert waren. Es kam zu einem Ausschlagen der proximalen Schrauben ( $\bullet$ Abb. 2b), die bei Dislokation der Fragmente weiterhin in der Platte verriegelt waren. Die Instabilität der Osteosynthese verursachte einen zusätzlichen Knochendefekt. Die erneute Osteosynthese wurde mittels winkelstabilem Implantat und als Verbundosteosynthese durchgeführt.

\section{Frakturheilungsstörung mit sekundärer Implantatdeformierung/ Plattenbruch}

Fall 3. Eine distale Femurfraktur wurde mit einem LISS-Implantat versorgt, das für eine angestrebte funktionelle Nachbehandlung nicht geeignet ist. Die spezifische Implantatwahl war somit nicht korrekt erfolgt. Unter Belastung kam es zu einer Deformierung des Implantats mit Korrekturverlust. Die Reosteosynthese wurde mit längerem LISS-Implantat durchgeführt. Bei ausbleibender Frakturheilung kam es zum erneuten Implantatversagen mit Plattenbruch. Nach erneuter Reosteosynthese mit einem LISS-Implantat - auch hier ohne Spongiosaplastik - kam es zur Ausheilung der Fraktur (- Abb. 3). 


\section{Zusammenfassung $\cdot$ Abstract}

Tabelle 1

\section{Lokalisation der Implantate}

\begin{tabular}{|c|c|c|}
\hline Lokalisation & Implantat & Anzahl \\
\hline \multirow[t]{3}{*}{ Oberarm } & $\operatorname{LCP} 3,5$ & 48 \\
\hline & LCP 5,0 & 11 \\
\hline & $\begin{array}{l}\text { Metaphysen- } \\
\text { platte }\end{array}$ & 4 \\
\hline \multirow[t]{2}{*}{ Unterarm } & LCP 3,5 & 50 \\
\hline & Radiusplatte & 181 \\
\hline \multirow[t]{3}{*}{ Oberschenkel } & LCP 5,0 & 10 \\
\hline & $\begin{array}{l}\text { Metaphysen- } \\
\text { platte }\end{array}$ & 1 \\
\hline & LISS & 24 \\
\hline \multirow[t]{5}{*}{ Unterschenkel } & LCP 3,5 & 18 \\
\hline & LCP 5,0 & 12 \\
\hline & LISS & 19 \\
\hline & Torno-Fix & 9 \\
\hline & $\begin{array}{l}\text { Metaphysen- } \\
\text { platte }\end{array}$ & 20 \\
\hline \multirow[t]{2}{*}{ Sprunggelenk } & LCP 3,5 & 36 \\
\hline & LCP 5,0 & 1 \\
\hline \multirow[t]{2}{*}{ Klavikula } & $\mathrm{LCP} 3,5$ & 17 \\
\hline & Radiusplatte & 1 \\
\hline \multirow[t]{2}{*}{ Wirbelsäule } & LCP 5,0 & 3 \\
\hline & Ventro-Fix & 1 \\
\hline \multirow[t]{2}{*}{ Becken } & LCP 3,5 & 2 \\
\hline & LCP 5,0 & 9 \\
\hline Kalkaneus & Kalkaneusplatte & 14 \\
\hline Gesamt & & 491 \\
\hline
\end{tabular}

Tabelle 2

\section{Komplikationen}

\begin{tabular}{|c|c|c|}
\hline & $\mathbf{n}$ & $\%$ \\
\hline Gesamt & 491 & 100 \\
\hline Komplikationen & 28 & 5,7 \\
\hline •Infekt & 7 & 1,4 \\
\hline $\begin{array}{l}\text { - Operationstechnisches } \\
\text { Implantatversagen }\end{array}$ & 7 & 1,4 \\
\hline $\begin{array}{l}\text { - Achsfehlstellung } \\
\text { sekundär mit Implantat- } \\
\text { deformierung }\end{array}$ & 7 & 1,4 \\
\hline - Achsfehlstellung primär & 4 & 0,8 \\
\hline - Schraubenfehllänge/-lage & 3 & 0,6 \\
\hline
\end{tabular}

\section{Implantatunabhängiger Korrekturverlust}

Im Rahmen einer prospektiven Studie wurde der Korrekturverlust nach Versorgung distaler Radiusfrakturen mit winkelstabiler Radius-T-Platte bei 100 Patienten ermittelt [10]. Das Ergebnis zeigte, dass
Trauma Berufskrankh $2005 \cdot 7[$ Suppl 1]:S65-S68

DOI 10.1007/s10039-004-0956-8

๑) Springer Medizin Verlag 2004

\section{F.-O. Reiter · T. J. Hockertz · A. Gruner · H. Reilmann}

\section{Lassen sich Korrekturverluste bei winkelstabilen Osteosynthesen vermeiden?}

\section{Zusammenfassung}

Die Vorteile der winkelstabilen Implantate liegen in der Erhöhung der axialen Stabilität, dem geringen Periostkontakt und besserer Festigkeit im vorgeschädigten Knochen durch das Schraubendesign. Einsatzmöglichkeiten finden sich u. a. im Bereich der langen Röhrenknochen, der Wirbelsäule und des Beckens. Gelenk- und gelenknahe Frakturen sowie Problemfrakturen wie periprothetische Frakturen können versorgt werden. Ein sekundärer Korrekturverlust lässt sich nicht immer vermeiden. Dabei müssen operationstechnische Fehler,

wie falsche Implantatwahl mit Folge des Implantatversagens, Frakturheilungsstörung mit Implantatdeformierung und systemunabhängiger Korrekturverlust unterschieden werden. Eine Minimierung des Korrekturverlusts ist durch eine kompetenzbasierte Instruktion in das jeweilige System zu erreichen.

\section{Schlüsselwörter}

Sekundärer Korrekturverlust .

Winkelstabile Implantate · LISS · LCP

\section{Can correction losses in fixed-angle osteosynthesis be avoided?}

\section{Abstract}

The advantages of fixed-angle implants are due to the design of the screws used, which results in increased axial stability, only slight periostal contact, and better seating of the implant in bone that is already damaged. These implants can be used, for example, in the long bones, the spine and the pelvis. They can be applied for the treatment of joint fractures and fractures in the vicinity of joints, and also for the treatment of such problem fractures as those close to prosthetic implants. It is not always possible to avoid secondary correction loss. It is im- portant to differentiate technical surgical errors, such as selection of the wrong implant with consequent implant failure, and impaired fracture healing with deformation of the implant from correction loss that is not dependent on the implant system at all. A sound introduction to the use of the system to be applied can keep correction loss to a minimum.

Keywords

Secondary correction loss Fixed-angle implants · LISS · LCP 

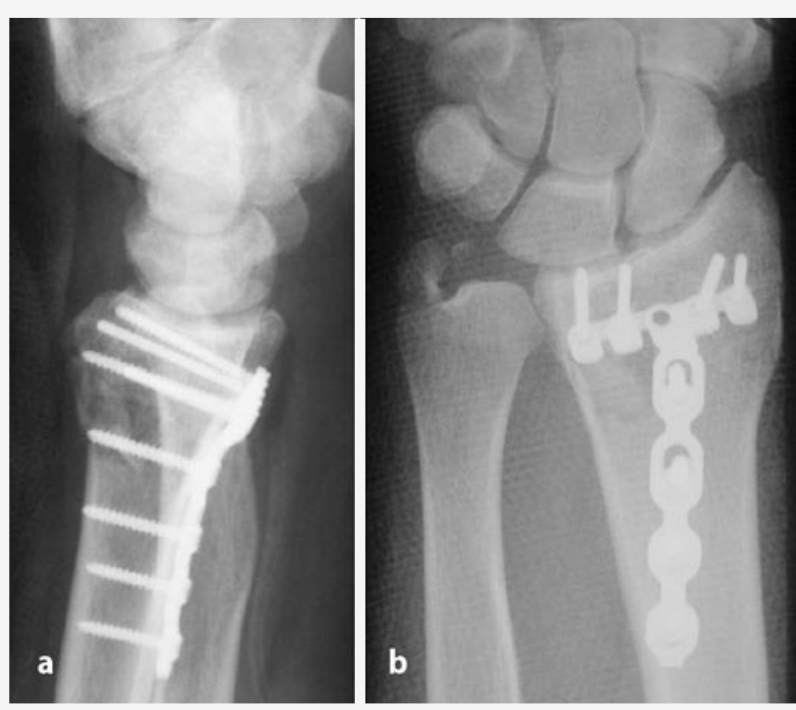

Abb. $4<$ Korrekturverlust distaler Radius

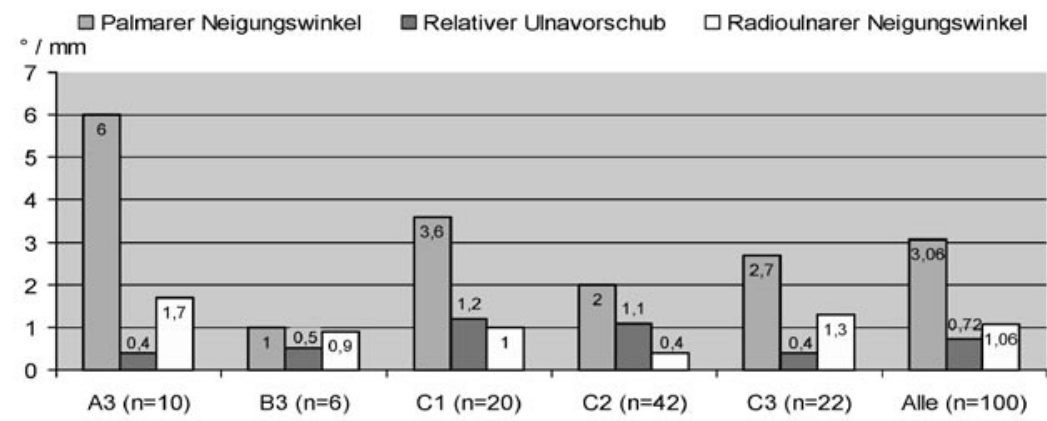

\section{Abb.5 $\Delta$ Korrekturverlust bei winkelstabiler Radius-T-Platte}

auch bei korrekter Osteosynthese ein implantatunabhängiger Korrekturverlust stattfindet, der auf spongiösen Knochen und damit verbundene Sinterung zurückzuführen ist. Dabei ist vorrangig der palmare Neigungswinkel betroffen. Auf alle Nachuntersuchten betrug der Korrekturverlust beim palmaren Neigungswinkel $3,06^{\circ}$, beim radioulnaren Neigungswinkel $1,06^{\circ}$. Der relative Ulnavorschub war o,72 mm (• Abb. 4, • Abb. 5).

\section{Fazit für die Praxis}

1. Korrekturverluste treten auch bei winkelstabilen Implantaten auf.

2. Hauptursache sind technische Fehler mit unzureichender Implantatverankerung im strukturschwachen Knochen.

\section{Korrespondierender Autor}

\section{Dr. F.-O. Reiter}

Unfallchirurgische Klinik, Städtisches Klinikum, Holwedestraße 16, 38118 Braunschweig E-Mail: Finn.Reiter@gmx.de

\section{Interessenkonflikt: Keine Angaben}

\section{Literatur}

1. Badke A, Kaps H-P (2004) Erste Erfahrungen mit winkelstabilen Implantaten an der Brust- und Lendenwirbelsäule. Trauma Berufskrankh [Suppl 1] 6: 41-43

2. Frigg R (2003) Development of the LCP. Injury [Suppl 2] 34: 6-10

3. Gautier E, Sommer C (2003) Guidelines for the clinical application of the LCP. Injury [Suppl 2] 34:6376

4. Gruner A, Hockertz TJ, Kleine B et al. (2001) Versorgung der Mehretagenfraktur der proximalen Tibia mit/ohne Gelenkbeteiligung durch geschlossene Reposition und Stabilisierung mit dem Less Invasive Stabilisation System - LISS (PLT). Hefte Unfallchirurg 283: 117-119
5. Gruner A, Hockertz TJ, Reilmann H (2003) Alternative Verfahren bei distaler Femurfraktur - Minimalinvasive Plattenosteosynthese. Trauma Berufskrankh [Suppl 1] 5:42-46

6. Hockertz TJ, Gruner A, Reilmann H (2001) Die Versorgung von peri- und interprothetischen Oberschenkelfrakturen mit dem LISS DF. Hefte Unfallchirurg 283: 360-361

7. Höntzsch D (2004) Erste Erfahrungen mit winkelstabilen Implantaten. Untere Extremität: distaler Femur, proximale und distale Tibia. Trauma Berufskrankh [Suppl 1] 6: 27-31

8. Kinzl L, Arand M, Einsiedel T (2004) Erste Erfahrungen mit winkelstabilen Implantaten am Fersenbein. Trauma Berufskrankh [Suppl 1] 6: 32-34

9. Matschke S, Kruhl Ch, Wentzensen A (2004) Erste Erfahrungen mit winkelstabilen Implantaten. Distale Radiusfraktur. Trauma Berufskrankh [Suppl 1] 6: 19-26

10. Sakhaii M, Groenewold U, Klonz A et al. (2003) Ergebnisse nach palmarer Plattenosteosynthese mit winkelstabilen T-Platten bei 100 distalen Radiusfrakturen. Eine prospektive Studie. Unfallchirurg 106: $272-280$

11. Schandelmaier P, Stephan C, Krettek C et al. (2000) Distale Femurfrakturen. Unfallchirurg 103: 428436

12. Sommer Ch, Gautier E, Müller M et al. (2003) First clinical results of the locking comression plate (LCP). Injury [Suppl 2] 34: 43-54

13. Südkamp NP (2004) Erste Erfahrungen mit winkelstabilen Implantaten. Obere Extremität: subkapitale Humerusfraktur. Trauma Berufskrankh [Suppl 1] 6: 13-18

14. Vock B, Matschke S, Grützner PA et al. (2004) Erste Erfahrungen mit winkelstabilen Implantaten an der Halswirbelsäule. Trauma Berufskrankh [Suppl] 6: $35-40$

15. Wagner $M$ (2003) General principles of the clinical use of the LCP. Injury [Suppl 2] 34: 31-42 